\title{
Implementation of Screening, Brief Intervention, and Referral to Treatment (SBIRT) by Nurses on Acute Care Units: A Qualitative Descriptive Study
}

Kelli Thoele, Claire Burke Draucker, Robin Newhouse

\begin{abstract}
Background Screening, Brief Intervention, and Referral to Treatment (SBIRT) is an evidencebased intervention for patients with substance use disorders, but this intervention is underutilized. Little is known about the implementation of SBIRT in acute care facilities. The purpose of this study is to describe implementation of SBIRT by nurses in acute care hospitals.

Methods A qualitative descriptive design was used for this study. Registered nurses who agreed to participate in the study completed a 1:1 interview using a semi-structured interview guide. Interviews were audio recorded and transcribed and then data were analyzed using qualitative content analysis.
\end{abstract}

Results When implementing SBIRT in an acute care setting, participants identified several factors that affect implementation. Some nurses felt that it is "one more thing to do" but other nurses feel that it was a "good, simple" screening tool that does not take long to do and can "plant the seeds of change" for patients. Additionally, participants mentioned barriers and facilitators related to the patients' responses to SBIRT and organizational factors.

Conclusions This study identified several barriers and facilitators to SBIRT implementation related to the nurses, patients, and organization. By understanding the factors that influence implementation, healthcare providers can develop strategies to support effective implementation of SBIRT.

This is the author's manuscript of the article published in final edited form as:

Thoele, K., Draucker, C. B., \& Newhouse, R. (2020). Implementation of screening, brief intervention, and referral to treatment (SBIRT) by nurses on acute care units: A qualitative descriptive study. Substance Abuse, 1-10. 


\section{Keywords}

Hospitals; nurses; Screening, Brief Implementation, and Referral to Treatment (SBIRT), Substance-related disorders; Substance-use disorders; Systems implementation

\section{Word Count}

7005 (main manuscript), 8073 (including abstract and references)

\section{Disclosure Statement}

The authors have no conflicts of interest. 


\section{Introduction}

Substance use disorders are prevalent and are associated with a variety of negative health outcomes and high financial cost for society. Worldwide, 18.5 million people have alcohol use disorder, 35 million people have a drug use disorder, and 1.1 billion people smoke tobacco. ${ }^{1,2,3}$ Misuse of alcohol and other drugs is associated with disorganized thinking, memory loss, mental health problems, cardiomyopathy, hypertension, liver disease, pancreatitis, kidney disease, infectious disease, and cancer. ${ }^{4,5}$ Each year, more than 10 million people worldwide die due to tobacco, alcohol, or drug use. ${ }^{6}$ Moreover, substance use is also associated with fetal alcohol syndrome, neonatal abstinence syndrome, intimate partner violence, and crime. ${ }^{7}$ The direct and indirect costs of substance abuse are estimated to be $\$ 740$ billion annually in the United States. ${ }^{8}$

Due to the serious consequences associated with substance use, healthcare providers should assess for misuse of alcohol and other drugs by patients and intervene when appropriate. Providers can be aided in this process by using Screening, Brief Intervention, and Referral to Treatment (SBIRT). According to the Substance Abuse and Mental Health Services Administration, SBIRT is "an evidence-based practice used to identify, reduce, and prevent problematic use, abuse, and dependence on alcohol and illicit drugs." ${ }^{9}$ SBIRT can be administered by healthcare providers in a variety of settings ${ }^{10,11}$ and includes three components. First, the provider uses a validated tool (e.g., Alcohol Use Disorders Identification Test, Drug Abuse Screening Test) to screen patients for risky substance use. Next, based on the results of the screening and clinical judgment, the provider may provide a brief intervention (e.g., motivational interviewing) to address substance use. Lastly, providers refer individuals to treatment when appropriate. 
SBIRT can be particularly useful in hospitals. Approximately 1 out of every 7 hospitalized patients have a substance use disorder in the United States. ${ }^{12}$ Nurses, the largest sector of healthcare providers in hospitals,${ }^{13}$ are well positioned to use SBIRT. Nurses routinely assess patients and use validated screening tools to identify patients who may need interventions. In addition, nurses are trained in therapeutic communication and are thus poised to learn techniques such as motivational interviewing. In addition, they regularly collaborate with members of the interdisciplinary healthcare team and thus can help facilitate and coordinate referrals to treatment. Moreover, patients report feeling comfortable discussing substance use with nurses. ${ }^{14}$

Although SBIRT is an evidence-based intervention, it is underutilized in healthcare settings. Research indicates that healthcare providers do not consistently use a validated tool to screen for substance use, and the majority of patients who use substances do not receive optimal treatment. ${ }^{15,16}$ More information is therefore needed on the implementation of SBIRT. Implementation is defined as "active and planned efforts to mainstream an innovation within an organization." ${ }^{\text {"17, p. } 582}$ The process of implementation includes adoption (the decision to use the intervention) and sustainment (continued use of the intervention). ${ }^{17,18,19}$ Studies evaluating implementation of SBIRT in various settings have been conducted, ,e.g. $20,21^{\text {but evidence }}$ regarding implementation of SBIRT by nurses in acute care settings, such as hospitals, is limited. The purpose of this study is to describe the implementation of SBIRT by direct care nurses employed on a hospital nursing unit that is in the process of implementing SBIRT.

\section{Methods}

\section{Design}


A qualitative descriptive design was used for this study. Based on textual data provided by participants, this approach is used to provide a straightforward account of a phenomenon in everyday language. ${ }^{22,23}$ In health services research, qualitative description can be used to obtain the perspectives of a variety of stakeholders about a system change. ${ }^{24,25}$ In qualitative descriptive research, purposive sampling is often used to identify participants who can provide the most useful information. ${ }^{22}$ Participant accounts are obtained through semi-structured interviews, direct observation, or examination of documents. ${ }^{22,23}$ Data often are analyzed using qualitative content analysis, and participants' own words are often used to describe events. ${ }^{22,23} \mathrm{~A}$ low level of interpretation is used so that the findings remain close to participant accounts. The product of qualitative description is a comprehensive summary of the phenomenon of interest that answers a specific practice or policy question. ${ }^{22}$ As the purpose of this study to describe in a straightforward manner the everyday experiences of the implementation of SBIRT by nurses providing direct care, relying heavily on their own words, qualitative description was determined to be the most appropriate method. The COREQ qualitative reporting guidelines were used to inform the writing of this manuscript. ${ }^{26}$

\section{Setting and Sample}

\section{Parent study}

This qualitative study supplements a cluster randomized implementation study examining the implementation of SBIRT at fourteen acute care hospitals in a healthcare system in the Midwest of the United States (referred to as the parent study). The hospitals were stratified by facility type and then randomized into an intervention group or a usual care wait-list control group. ${ }^{26}$ The chief nursing officer at each facility selected one medical-surgical nursing unit to participate in the parent study and one site coordinator to lead efforts at each facility. Each 
medical-surgical nursing unit selected for participation in the study provided care for medical and/or surgical patients who required hospitalization but did not require admission to an intensive care unit. The site coordinators in the parent study received training and on-going support to implementation of SBIRT. ${ }^{27}$ All of the hospitals in the intervention group began training in January 2018 and started using SBIRT between April and July 2018. Each site coordinator worked with leaders at each hospital to adapt the SBIRT intervention. For example, the majority of nurses on each study unit were trained to use a validated tool to screen each patient for risky substance use within 24 hours of admission. Leaders at some hospitals trained a group of nurses to complete the brief intervention, and leaders at other hospitals determined that the social workers would complete the brief intervention component of SBIRT if indicated. The referral to treatment was completed by nurses, social workers, physicians, or other healthcare providers at each hospital, and a referral included either a recommendation to seek treatment after hospital discharge or a referral to a specific outpatient treatment facility upon discharge. After implementation of SBIRT, there were significant improvements in the percentage of patients screened for alcohol and drug use with a validated tool from $1 \%$ of patients to $40 \%$ of patients $(\mathrm{p}<0.001){ }^{28}$

\section{Current study}

The research team for the study reported here (referred to as the current study) included the primary investigator, who is a doctoral nursing student and research assistant for the parent study (KT); an expert in implementation science and lead investigator of the parent study (RN); and an expert in qualitative research (CD).

The investigators invited the chief nursing officers at all seven hospitals in the intervention group of the parent study to invite registered nurses working on the participating 
medical-surgical units to be interviewed, and six chief nursing officers agreed. The participating institutions included 3 critical access hospitals, 2 academic health centers, and 1 community hospital. The average hospital bed size was 247 beds (range $=15$ to 858 ), and the average nursing unit bed size was 25 (range $=15-36)$. Four of the hospitals were recognized by the American Nurses Credentialing Center as either a Magnet ${ }^{\circledR}$ facility or a Pathway to Excellence ${ }^{\circledR}$ organization.

Nurses who were scheduled to work at least 20 hours per week, were employed at least six months on the medical-surgical unit participating in the parent study, and had been trained in either the screening or brief intervention components of SBIRT were eligible to participate. These criteria ensured that participating nurses were familiar with the implementation of SBIRT.

To recruit participants by purposive sampling, investigators obtained a list of nurses who met criteria from the unit manager or SBIRT site coordinator at each facility. Eligible nurses were divided in groups according to the type of SBIRT training they had received: Screening Only or Screening/Brief Intervention. Nurses were randomly selected from each group in proportion to the numbers of nurses on each unit who had received each type of training. For example, if $75 \%$ of the nurses on a unit were trained in Screening/Brief Intervention, then $75 \%$ of nurses recruited for the current study were drawn from this group. The investigators provided a one-page flyer about the study to the nursing manager for dissemination to all nursing staff, and recruitment emails were sent to selected nurses, along with a study information sheet. If a nurse did not respond to the initial recruitment email, a second and final recruitment email was sent one week later. Recruitment continued until 4 participants from each site completed interviews or until all eligible nurses had been contacted. This recruitment technique and sample 
size target was to ensure representation from multiple nurses from each facility to account for variation among facilities.

\section{Data Collection}

Two of the investigators (KT and CD) developed a semi-structured interview guide comprised of fifteen open-ended questions (see Appendix A). The development of the guide was informed by the theoretical domain framework ${ }^{29}$ that identifies several domains (e.g. knowledge, beliefs, context, influences) that explain behavior change in the implementation of evidence-based interventions. The interview guide started with introductory questions about familiarity with SBIRT and initial exposure to SBIRT implementation, followed by questions about the decision to use SBIRT (adoption), efforts to mainstream SBIRT into practice (implementation) and continued use of SBIRT (sustainment). Sample questions were as follows: (a) What did you think of SBIRT when you first heard about it?, (b) How do you decide if you will use SBIRT when you admit a patient?, (c) Tell me about a time you used SBIRT and if it went well/did not go well?, and (d) Do you intend to continue to use SBIRT in your practice?. The qualitative expert $(C D)$ provided feedback on the primary investigator's interview technique after a practice interview with a graduate nursing student and the first two transcripts were completed.

The primary investigator (KT) conducted one-to-one phone interviews with participants using the semi-structured guide and employed additional follow-up questions as needed to fully understand each participant's perspective. The participants were asked to be at a private location during the phone interviews. Most participants completed the interview at home. In a few instances, family members were present in the home at the time of the interview, but this did not distract from the conversation. Interviews were audio recorded and professionally transcribed. 
The interviewer recorded field notes in Microsoft Word at the completion of each interview. All interviews were conducted between January 2019 and June 2019.

Although the interviewer (KT) was employed within this healthcare system at one time, she had not had prior contact with any of the participants. The interviewer's credentials and status as a PhD student were included in the recruitment emails and study flyer.

\section{Data Analysis}

Data were analyzed using conventional qualitative content analysis and inductive category development. ${ }^{30}$ Data analysis was an iterative process and began after the first interview. First, the primary investigator (KT) compared each audio recording with the transcripts to check for accuracy and read each transcript multiple times to understand the participants' experiences with SBIRT holistically. Next, the primary investigator (KT) extracted text units from each transcript. Text units are words, phrases, or sentences that capture a single coherent point made by the participants. Each text unit was assigned a code (a short phrase) to capture the essence of the text unit. $^{31}$

All codes were placed into a table organized into cells created by placing the participants' identification number on the vertical axis and the interview questions on the horizontal axis (e.g., Participant $001 \mathrm{X}$ initial thoughts about SBIRT). All codes in each column were reviewed and condensed into categories by the primary investigator (KT) and reviewed by the qualitative expert (CD). ${ }^{32}$ Through discussion and consensus of all team members (KT, RN, CD), the categories were finalized, labeled, and described. All analytic activities were facilitated with the use of Microsoft Word software. Because the transcripts were verified for accuracy by the primary investigator (KT) and a low interpretive analysis was used, study participants were not asked to verify the accuracy of the transcripts or provide feedback on the findings. ${ }^{33}$ 


\section{Ethical Considerations}

Indiana University Institutional Review Board approval was obtained from the research team's institution (\#1809264693), and administrative approval was obtained from each participating hospital. Prior to interviews, the investigator reviewed the study information sheet and answered all questions about participation in the study. Each participant verbally agreed to participate in the study.

\section{Results}

Seventy-one nurses were invited to participate in the study, 21 agreed to participate, and 18 met eligibility criteria and were interviewed. The majority of participants were women, worked the day shift, had a bachelor's degree, and had been trained in both the screening and brief intervention (Table 1). The average length of the interviews was 21.1 minutes (range $=8$ to 36 minutes). After the completion of 18 interviews, the investigators concluded that the interviews had yielded sufficiently rich information to address the study aims.

The participants described how they implemented SBIRT primarily by discussing factors that got in the way of their use of SBIRT or that helped them use SBIRT. The team thus determined that the findings best took the form of a listing and description of barriers to and facilitators of the implementation of SBIRT from the perspectives of direct care nurses charged with using SBIRT in their day-to-day practices. The team also determined that the barriers and facilitators could be divided into three domains: (a) nurses' attitudes and beliefs about SBIRT, (b) organizational factors related to implementing SBIRT, and (c) patients' responses to SBIRT. The barriers and facilitators are listed in Table 2 and described below.

\section{Barriers to Implementation of SBIRT}


When discussing their experiences with SBIRT, the participants discussed a number of factors that interfered with their day-to-day use of SBIRT and factors that made it arduous or bothersome for them. These factors were related to their own attitudes and beliefs about SBIRT, factors in their organization that influenced the implementation of SBIRT, and patients' responses to SBIRT.

\section{Nurses' attitudes and beliefs about SBIRT}

The participants expressed seven attitudes or beliefs that suggested they were critical or skeptical about the use of SBIRT. First, upon initial exposure to SBIRT, several participants felt that SBIRT was just "one more thing" to do. Because they already were required to get a good deal of information when admitting patients, SBIRT only added to this process. One participant said that her initial impression of SBIRT was that it was "one more thing we have to do at the admission, which is already pretty lengthy." Other participants expressed being very busy generally and stressed that any new responsibilities, such as SBIRT, added to their workload burden. Second, some participants were troubled that nurses and other healthcare providers cannot observe the long-term outcomes of SBIRT and thus were uncertain if SBIRT "makes a difference" for patients. Some participants were doubtful that patients would follow through with referrals they had been given. One stated, "And if they did a referral, if they needed a referral, what happened after these four walls? What happened afterwards? ... So, what impact can we have outside of these four walls? I don't know how you'd measure that." Third, some participants noted it is uncomfortable to ask patients about substance use and challenging not to seem judgmental. These participants were concerned SBIRT puts patients "on the spot." Participants were especially reluctant to ask about substance use if they felt that it might harm 
their relationship with a patient and impede their ability to care for the acute needs of the patient. One participant stated,

You know what really bothered me, was that this is their first impression of me... I'm going to take care of you, I'm trying to build a trusting relationship, and I'm asking you these questions. It was just really uncomfortable because that's their first impression of me and my unit was asking those questions and them thinking the judgements that they think and their perception. It was very uncomfortable.

Fourth, some participants suggested that SBIRT, especially the motivational interviewing component, was not a nursing function but rather would be more appropriately provided by social workers. The participants further suggested that social workers are better situated to provide the intervention because they could follow-up with patients whereas patients could have a different nurse every day. For example, one participant stated,

I do think it [brief intervention] would tend to work better with somebody who is going to see them [the patient] consistently throughout the stay, like social workers...the same thing coming from the same person two or three days is a lot different than the person who just started your IV [intravenous catheter].

Fifth, a few participants, while they saw the value of SBIRT to screen for acute problems like withdrawal in the hospital, argued that an acute care unit is not the best setting to address a chronic problem with substance use. When asked if she would continue to use SBIRT, one participant replied,

Just to keep my patients safe in the hospital? Yes. I want them to go home and not use alcohol, but that requires outpatient therapy. But to keep our patients safe while in the hospital? Yes, I'll continue to screen them. 
Sixth, a few participants felt that SBIRT was unnecessary because healthcare providers are often already aware of the patients' histories with substance abuse, especially if they are frequently admitted. For example, the participants argued that if an admitting physician or emergency department provider already asked about substance use, nurses' use of SBIRT would not provide new information. One participant said, "Usually when a patient comes in [who] is intoxicated or has a drug problem, a lot of times you already know because they tell the ER [emergency room]." Seventh, a few participants felt they did not have the knowledge or skills to intervene if the patient screened positive on the screening tool. These participants wanted to help patients address their substance use but felt unprepared to provide the intervention or referral. For example, one participant stated, "I feel like okay, well today we opened the wound, but we didn't do anything. So, it's frustrating at times. I'm doing it [screening], but I don't know why, because I've got no intervention for you."

\section{Organizational Factors}

Participants identified six organizational factors that they viewed as barriers to the use of SBIRT. First, some participants mentioned that is difficult to maintain competency in the brief intervention because they rarely had the opportunity to use it. One pointed out that if a nurse works three shifts a week and only admits a few patients each week, it's possible to go several weeks without admitting a patient who requires a brief intervention. One stated that motivational interviewing is

a muscle you have to work though, and that's the main problem with SBIRT. It's so rare that we run across anybody who would need an intervention, any kind of intervention for substance use. It's a use it or lose it skill, you know? 
Second, some participants worried that no one assessed how they were implementing SBIRT or provided feedback. As one participant stated, "There was no follow-up ... or any data that we can see how well we were doing. There was no feedback ... no follow-up ... no, 'Hey, you did this, you should have done this.",

Third, a few participants noted that SBIRT was not integrated into the electronic medical record and therefore more difficult to incorporate into their workflow. One participant said, "We don't have this included as part of our admissions in Cerner [Cerner PowerChart ${ }^{\circledR}$, the electronic medical record used at this facility]. I think a lot of time it's just an honest 'I forgot to do that kind of thing."' Fourth, a few participants identified inadequate training as a barrier to SBIRT implementation. One participant stated, "Shift coordinators and charge nurses went and had a presentation regarding [SBIRT]. It wasn't well structured though. It seemed like it was vague...the people giving the presentation had some type of questions themselves and couldn't answer the questions we had." Fifth, a few participants cited competing priorities as a barrier to the use of SBIRT. When admitting a patient and caring for acute problems, addressing substance use was not a top priority. For example, one participant stated "In an admission, you're focused on getting all the details right about their home meds...going through orders, making sure all the ducks are in a row for this person...I hate to say it, but [SBIRT], it's a lesser priority." Sixth, a few participants stated that lack of adequate resources in the community was a barrier to the use of SBIRT. For example, some communities do not have adequate treatment centers or enough programs to refer patients. One participant stated that her community "lacks rehab support for patients. I mean you can't conquer the opioid and meth addiction without that support environment outside of the hospital."

Patients'Responses 
Participants identified five barriers related to patients' responses to SBIRT. First, some participants felt that patients hide substance use. These participants noted that patients may be reluctant to admit to substance use or the severity of the problem, and this limits the nurse's ability to address substance use. One participant stated, "There's no incentive for them to tell the truth and make themselves look worse...You're starting out with information that may or may not be accurate." Second, some participants noted that patients might not be ready to change their behavior even if they chose to disclose their substance use. One participant stated,

I just don't feel like they're open at that time to change, because people like to drink alcohol. That's their mindset, and if they don't want to change it, then I'm not going to influence them in the two days they're in the hospital.

Third, a few participants stated that patients are reluctant to open up about substance use until a trusting nurse/patient relationship has been established. For example, one participant said “They first don't want to trust you. They try to manipulate, and then you realize they're like 'I'm here for your best interests, I want to make you better. I want to help you, see you succeed."' Fourth, a few participants felt that patients were angry that nurses were asking about substance use or that patients felt judged. One participant stated that "Smoking and drinking alcohol, those are legal activities for adults to do, so usually people are pretty honest...but I feel like the drug question, you're going to get people that get angry." Fifth, a few nurses identified that SBIRT was more difficult to complete when patients were tired or in pain, and yet if the nurses delayed SBIRT until the patient was ready, it might not be completed. This was especially likely if patients were admitted on night shift or had uncontrolled pain. For example, a night shift nurse stated, "At night, a lot of times folks just want to get it over with and go to sleep, so it might not be the best time to say, 'Let's have a talk about your bad habits."' 


\section{Facilitators to the Adoption and Implementation of SBIRT}

The participants also discussed a number of factors that encouraged their use of SBIRT in their day-to-day practices or contributed to their plans to continue to use it in the future. The facilitators included their own positive attitudes and beliefs, knowledge, and skills related to SBIRT; factors in their organization that promoted the use of SBIRT; and patients' constructive responses to SBIRT.

Nurses' attitudes, beliefs, skills, and knowledge related to SBIRT

Participants expressed nine factors that facilitated the use of SBIRT in their practice based on their own responses to SBIRT. First, most participants indicated SBIRT was embraced by nurses who were comfortable asking sensitive questions and could ask open-ended questions in a conversational and non-judgmental tone. One participant stated,

You have to be able to be a good communicator, and you have to not be judgmental of their [patients'] situation... ask the questions in a nice tone... Just show some concern, because obviously we're concerned about them, but you want them to feel you're concerned about it [substance use], and not just another question, a ho-hum thing. Second, most participants already felt confident identifying problems with substance use and intervening to help patients, so SBIRT was an extension of these skills. These participants noted that SBIRT could reveal substance use that may not be identified by just asking patients how much they drank alcohol or used drugs. One participant stated

We deal with most of that type of population all the time anyway, so it's kind of like why do we have to specifically ask these questions? But after starting to use it, you're actually kind of shocked by the amount of people that could actually use some, I guess, 
reference or direction. Some people you're like, 'Oh, maybe this could be something that we could help you with that is not completely related to your acute problem.'

Third, several participants felt that SBIRT could point to the need for acute interventions such as ordering a nicotine patch or monitoring to prevent withdrawal during hospitalization. Several nurses credited SBIRT with alerting them to patients' risks for withdrawal, and, in fact, some told patients that that was the purpose of the screening. One participant said,

It's a good screening tool to help me prevent major withdrawal symptoms in the hospital. So in my mind, SBIRT is good for me to prevent the patient from going through withdrawal... so I can help them prevent their withdrawal symptoms. So I can see it coming.

Fourth, several participants felt that SBIRT expanded the nursing role while identifying problems amenable to nursing care. One participant stated, "I think that's just part of our role, to be proactive in helping to identify a problem and help get them the right information." Another participant had not heard of SBIRT prior to participating in the parent study, but when asked if she would continue to use SBIRT in her nursing practice, she said,

I honestly think, personally for me, I would be remiss in my duties as a nurse if I didn't do that [screening and brief intervention] because it is so simple... it doesn't even take two minutes to have that conversation, two minutes. If we can't give two minutes with a patient to have that conversation and provide the knowledge, then I honestly think I would be remiss in my duties as a nurse.

Fifth, some of the participants found SBIRT to be a useful tool that could 'plant the seed' for change. These participants felt that even if patients did not immediately decide to change their 
behavior, SBIRT could lay the groundwork for addressing their substance use. One participant stated,

I felt like I planted a seed in someone...I asked some of those questions about 'have you harmed your family'... and he had to answer that he had. The look on his face when that question was asked, I feel like that's where the seed was planted.

Sixth, some participants noted that SBIRT made conversations about substance use easier for them as the screening tool provided ready-made ways of asking about substance use in nonjudgmental and consistent ways and could be presented as part of a routine assessment. One participant stated, "It's made me calmer and easier about asking those questions. Just like your date of birth and all these other questions you have to ask, it's just made it easier ... to ask those particular questions for any patient." Seventh, some participants mentioned that the use of SBIRT is facilitated if nurses already have knowledge about addiction, stages of change, how to complete the brief intervention, and available resources in the hospital and in the community.

Eighth, a few participants appreciated that SBIRT included providing information about resources after hospitalization, such as social services or Alcoholics Anonymous, to patients. One participant revealed that nurses at her facility had compiled a list of community resources that she referred to as the "SBIRT files." The participant said,

I had a patient who had significant problems with meth, and I don't think she realized we actually have programs available in the community...I happened to be aware of other services in the community, so I provided her with the information about methamphetamine abuse the we provide through the SBIRT files. 
Ninth, a few participants noted that the SBIRT screening questions were easy to ask and did not take long to complete. One participant stated "The form is really nice, easy to use, and it can be quick...it's very easy to use.”

\section{Organizational Factors}

Participants mentioned six organizational factors that facilitated the implementation of SBIRT. First, most participants noted that SBIRT was easier to use in their practices if it was incorporated into an established routine on their unit such as the standard admission process. SBIRT was more likely to be completed if the screening tool was automatically added to the admission packet or checklist. As one participant stated,

I just tried to integrate it as part of my admission. I just got in the habit of asking those questions with part of my admission spiel, and I actually made an admission checklist for my unit... a sheet that makes it nice and easy for the nurses, especially the newer ones to go okay, these are the things I need to focus on.

Second, several participants noted their use of SBIRT had become a routine practice because it was expected for all nurses on their unit. One participant, when asked how she decides if she is going to use SBIRT, stated that she does not "consciously" make a decision about it. She said, "We do it with everyone now." Third, several participants stated that SBIRT is easier to use if there is a clear and streamlined process on their unit that includes clear expectations about who completes it, a consistent place where required forms are kept, and easily available resources such as patient education materials and a list of patient resources. One participant who was only trained in screening stated, "The nurse that does the usual screening has to ask, 'Do you use alcohol.' If that's a yes, then you mark it and pass it to the charge nurse." Fourth, some of the participants noted that good initial training and ongoing support and coaching facilitated the 
implementation of SBIRT. For example, one participant recommended that future sites that implement SBIRT should provide good education to the people who are using it...like through a communications class or role-playing, or a demonstration. Just something that you can follow up with the people who are using it. Have them give you a situation where it worked or it didn't work, and what they can do to improve it.

Fifth, some of the participants stated that an enthusiastic "champion" was essential for the implementation of SBIRT. The site coordinators in the parent study served as SBIRT champions. They lead implementation efforts, provided initial training, developed implementation procedures, and adapted the intervention when needed. When discussing the site coordinator, one participant stated,

She has been very, very passionate about this, and she has put on a lot of in-services...on how to use the tool. And she also gave us these cards, so if you have it right here there and think 'Okay, well now I need to do a brief intervention and now I need to talk to the doctor.' Let's get a plan in progress.

Sixth, a few participants mentioned that the ability to adapt the intervention to the needs of their unit facilitated the use of SBIRT. Although the core components of SBIRT needed to remain the same, nurses at each facility could adjust the way that SBIRT was incorporated into practice at their facility. For example, nurses at some facilities created checklists or a process to facilitate hand-off or designated specific nurses to complete the brief intervention. A participant stated, "I think the [screening] form changed from the initial one to the one that we use now...the initial one that we used was a little bit more complicated."

\section{Patients' Responses}


Participants noted three patient responses to SBIRT that facilitated its use in practice. First, several participants stated that SBIRT works because patients are typically honest about substance use. One participant stated, "Most of the time people seem to be very honest and forthcoming about how much they either drink or use drugs. I've been kind of surprised a couple times." Second, some participants noted that SBIRT works better once a relationship has been established between the patient and nurse. One participant, who works on a unit with a patient population that often has a history of intravenous drug use and long hospitalizations, stated, We have patients that are there [on the unit] for a long time and they start trusting you. And then you're going to have to start asking these questions, and it's amazing the stuff that they tell you, since they've been there for a week and met you four out of those seven days... what they are willing to share then...social work issues that need to be addressed, that you wouldn't have expected that you needed to do. But now you're like okay, now it's coming together, and you get the full picture.

Third, some participants stated SBIRT is most successful when patients are willing to change their behaviors regarding substance use and accept available resources and take advantage of them. One participated stated, "You feel like it goes well when you have a patient that's ready for a change, and you can kind of match them with the resources that they need." Another participant, who worked on a unit that offered group therapy, described a patient who Started going to group, loved it. Her whole attitude changed once she gained knowledge of why she was an addict and what she was doing to herself. It gave her those skills that she needed to be able to fight the addiction.

\section{Discussion}


SUDs are prevalent in the United States and even more prevalent for people who are hospitalized in acute care units. As nurses aim to provide holistic care to patients, it is incumbent to provide evidence-based interventions, such as SBIRT, to screen for and treat SUDs. While SBIRT has been implemented successfully in primary care and emergency departments, its implementation in acute care settings is less established. This qualitative descriptive study was conducted to understand implementation of SBIRT by acute care nurses.

Participants identified several factors that hindered or enabled the implementation of SBIRT in their practices. Some of the barriers and facilitators to implementation of SBIRT revealed in our study are comparable to those found in other studies. In one of the first studies about implementation of SBIRT, for example, healthcare providers working in an emergency department identified several barriers to SBIRT that were also identified in our study. These barriers included a lack of time and referral resources, a lack of a role models, potential resistance from patients, and a lack of belief in the effectiveness of SBIRT. ${ }^{34}$ In a study of implementation of SBIRT in primary care, Hargraves et al. ${ }^{35}$ identified best practices for implementation, and these practices resonate with the results of our study. The best practices included having a practice champion, implementing SBIRT by an interprofessional team, clearly detailing the steps of SBIRT, offering ongoing training, aligning SBIRT with office flow, and integrating SBIRT into the electronic health record. ${ }^{35}$

Broyles et al. ${ }^{36}$ identified several barriers and facilitators anticipated by nurses who were planning to implement SBIRT in an acute care setting. First, Broyles et al. ${ }^{36}$ participants specified that SBIRT can be used to determine the risk of withdrawal. Our findings are consistent, indicating that SBIRT can be used to predict, plan for, and treat withdraw symptoms in acute care settings. Second, the participants in both our study and the Broyles et al. study ${ }^{36}$ 
cited issues of trust related to the implementation of SBIRT. Some participants believe patients are most likely to disclose substance use after developing a trusting relationship and that asking about substance use early on in an acute care setting can be an impediment to developing such trust.

While many of our study findings resonate with those of prior studies, several findings have not been given much prior consideration in the literature. The first is confusion about whether SBIRT is a nursing function. Some participants noted that social workers are better prepared to deliver a brief intervention. Another barrier not addressed previously is the logistics and timing of SBIRT. Nurses have difficulty conducting SBIRT when patients are admitted overnight or in acute distress. Our participants also mentioned several facilitators not extensively addressed in the literature. While participants noted that nurses who provide SBIRT in acute care settings will not see the long-term outcomes for patients, some felt that it was still worthwhile to provide SBIRT and 'plant the seed' for change during hospitalization. Another finding of our study that adds to the literature was that the implementation of SBIRT was facilitated when it was incorporated into a routine process in a user-friendly workflow.

\section{Strengths and Limitations}

There are several strengths and limitations to this study. The qualitative descriptive design yielded a robust list and description of barriers and facilitators to implementing SBIRT as identified by direct care nurses in day-to-day practice. Because the findings rely heavily on the participants' accounts of implementing SBIRT, the results can be used to develop strategies to improve implementation of SBIRT on acute care units. Another strength is that the participants worked in six different acute care facilities and represented a variety of ages, years of nursing experience, and shifts worked. One study limitation, however, is the potential for selection bias. 
The nursing units that participated in the parent study (and therefore in the current study) were selected by chief nursing officers and thus might represent units with higher capacity or inclination for change. In addition, only $25 \%$ of nurses who were contacted by the primary investigator (KT) agreed to participate in the current study. Nurses who refused participation may have been those who were less invested in SBIRT or those who might have been reluctant to discuss dissatisfactions with SBIRT despite the promise of confidentiality. Future research studies should implement SBIRT on all acute care units in a variety of facilities and obtain higher participation of direct care nurses in order to determine how varying unit and facility cultures related to substance use treatment and individual nurses' attitudes effect the use of SBIRT. Quantitative studies could then be aimed at measuring influencing factors and determining which ones are most associated with the successful implementation of SBIRT on acute care units.

\section{Implications}

Despite limitations, several practice implications can be drawn from the study findings. The list of barriers and facilitators can inform decisions about how to implement SBIRT on acute care units. Specifically, the findings have implications related to addressing nurses' attitudes and beliefs that hamper the implementation of SBIRT, addressing organizational factors that might impede the implementation of SBIRT, and understanding addiction and developing strategies to decrease patients' resistance to assessment and/or treatment of substance use.

Nurses concerns about SBIRT might be ascertained and addressed during initial training and then periodically through on-going supervision by SBIRT champions. For example, the concern that acute care nurses will not observe the long-term outcomes of SBIRT could be offset with a discussion of the notion of the importance of "planting the seed." Similarly, nurses' focus 
on SBIRT as a tool for only assessing risk for withdrawal during hospitalization could prompt a discussion of the broader goals of SBIRT as an intervention to address substance use as a chronic health problem. To counteract nurses' apprehensions that they are not best positioned to deliver SBIRT, champions can emphasize how nurses possess the basic communication and collaboration skills needed to implement the three components of SBIRT, and how more specialized skills, such as motivational interviewing or awareness of community resources available for referral, will be addressed in training.

The findings related to organizational factors that hinder or help acute care nurses' use of SBIRT have implications for administrators charged with implementing SBIRT. The findings indicate that SBIRT training needs to be conducted by instructors highly experienced in SBIRT and that on-going support, feedback, and supervision, including opportunities to refresh skills, is important. Because the results clearly reveal that nurses struggle with competing priorities and demands, their suggestions that SBIRT be integrated in the electronic medical record and be incorporated into their normal workflow should be considered when SBIRT is implemented on an acute care unit. Additionally, although the core components of SBIRT should remain the same, our findings indicate the benefit of adapting implementation of SBIRT to fit the context of the organization.

The findings regarding how patient factors influence the nurses' use of SBIRT also has implications for practice. Nurses should be provided education about addiction which addresses why patients might not be truthful about their substance use, may not be ready to disclose it, or might become angry if asked about substance use. SBIRT training and supervision can stress that these responses can be typical responses to addiction and do not preclude the use of SBIRT. SBIRT training should provide a variety of strategies to best address these responses. Moreover, 
our findings suggest that patient factors should drive when SBIRT is administered as it will not be effective when patients are in acute distress.

\section{Conclusion}

To provide holistic care to people admitted to acute care facilities, the implementation of SBIRT, an evidence-based intervention for substance use disorders, should be considered a best practice. This qualitative descriptive study included interviews with 18 direct care nurses who were expected to use SBIRT in their routine daily practices. Several barriers and facilitators that influenced their use of SBIRT were identified and described. The barriers and facilitators occurred in three domains: nurses' attitudes, beliefs, and skills; organizational factors, and patient responses. The findings can be used to guide SBRIT champions and facility administrators in improving the adoption, implementation, and sustainment of SBIRT on acute care nursing units.

\section{Acknowledgements}

This study was funded by Sigma Theta Tau International- Alpha Chapter.

\section{Declaration of Interest}

None 


\section{References}

1. World Health Organization. Global status report on alcohol and health 2018 https://apps.who.int/iris/bitstream/handle/10665/274603/9789241565639eng.pdf?ua=1\&ua=1. Accessed January 12, 2020.

2. World Health Organization. Prevalence of tobacco smoking. https://www.who.int/gho/tobacco/use/en/ Accessed January 12, 2020

3. United Nations. World drug report 2019: Executive summary. https://wdr.unodc.org/wdr2019/prelaunch/WDR19_Booklet_ 1_EXECUTIVE_SUMMARY.pdf Accessed January 12, 2020.

4. National Institute on Alcohol Abuse and Alcoholism. Alcohol's effects on the body. https://www.niaaa.nih.gov/alcohols-effects-body. Accessed September 25, 2019.

5. National Institute of Drug Abuse. Commonly abused drugs. https://d14rmgtrwzf5a.cloudfront.net/sites/default/files/nida_commonlyuseddrugs_final_ printready.pdf. Accessed September 25, 2019.

6. GBD 2017 Risk Factor Collaborators. Global, regional, and national comparative risk assessment of 84 behavioural, environmental and occupational, and metabolic risks or clusters of risks for 195 countries and territories, 1990-2017: A systematic analysis for the Global Burden of Disease Study 2017. The Lancet. 2018;392, 1923-1994. doi: $10.1016 / \mathrm{S} 0140-6736(18) 32225-6$

7. U.S. Department of Health and Human Services (HHS), Office of the Surgeon General. Facing addiction in America: The Surgeon General's report on alcohol, drugs, and health. Washington, DC:HHS. 
https://addiction.surgeongeneral.gov/sites/default/files/surgeon-generals-report.pdf. Accessed September 25, 2019.

8. National Institute on Drug Abuse. Trends \& statistics. https://www.drugabuse.gov/ related-topics/trends-statistics\#supplemental-references-for-economic-costs. Accessed August 30, 2019.

9. Substance Abuse and Mental Health Services Administration. SBIRT: Screening, Brief Intervention, and Referral to Treatment. https://www.integration.samhsa.gov/clinical-practice/sbirt. Accessed November 19, 2019.

10. Aldridge A, Linford R, Bray J. (2017). Substance use outcomes of patients served by a large US implementation of Screening, Brief Intervention and Referral to Treatment (SBIRT). Addict. 2017;112(Suppl. 2):43-53. doi:10.1111/add.13651

11. Substance Abuse and Mental Health Services Administration. About screening, brief intervention, and referral to treatment (SBIRT). https://www.samhsa.gov/sbirt/about. Accessed January 28, 2019.

12. Center for Health Information and Analysis. Behavioral health \& readmissions in Massachusetts acute care hospitals. Commonwealth of Massachusetts. http://www.chiamass.gov/behavioral-health-and-readmissions-inmassachusetts-acute-care-hospitals. Accessed September 25, 2019.

13. Bureau of Labor Statistics. Registered nurses have highest employment in healthcare occupations: anesthesiologist earn the most. https://www.bls.gov/opub/ted/2015/registered-nurses-havehighest-employment-in-healthcare-occupations-anesthesiologists-earn-the- 
most.htm. Accessed April 5, 2018.

14. Broyles LM, Rosenberger E, Hanusa BH, Kraemer KL, Gordon AJ. Hospitalized patients' acceptability of nurse-delivered screening, brief intervention, and referral to treatment. Alcohol Clin Exp Res. 2012;36(4):725-731. doi: 10.1111/j.1530-

0277.2011.01651.x Alcohol

15. Agley J, Carlson JM, McNelis AM, Gassman RA, Schwindt R, Crabb D, Vannerson J. 'Asking' but not 'screening': Assessing physicians' and nurses' substance-related clinical behaviors. Subst Use Misuse. 2018;53(11):1834-1839. doi:

$10.1080 / 10826084.2018 .1438806$

16. Substance Abuse and Mental Health Services Administration. (2019). Key substance use and mental health indicators in the United States: Results from the 2018 National Survey on Drug Use and Health (HHS Publication No. PEP19-5068, NSDUH Series H-54).

Rockville, MD: Center for Behavioral Health Statistics and Quality, Substance Abuse and Mental Health Services Administration. https://www.samhsa.gov/data/ Accessed August 30, 2019.

17. Greenhalgh T, Robert G, Macfarlane F, Bate P, Kyriakidou O. (2004). Diffusion of innovations in service organizations: systematic review and recommendations. Milbank Q. 2004;82(4):581-629.

18. Aarons GA, Hurlburt M, Horwitz SM. Advancing a conceptual model of evidence-based practice implementation in public service sectors. Adm Policy Ment Health .2011;38(1): 4-23. doi: 10.1007/s10488-010-0327-7 
19. Wisdom JP, Chor KHB, Hoagwood KE, Horwitz SM. Innovation adoption: A review of theories and constructs. Adm Policy Ment Health, 2014;41(4): 480502. doi:10.1007/s10488-013-0486-4.

20. O'Grady MA, Kapoor S, Kwon N, Morley J, Auerbach M, Neighbors C J. et al. Substance use screening and brief intervention: Evaluation of patient and implementation differences between primary care and emergency department settings. $J$ Eval Clin Pract. 2019;25(3):441--447. doi:10.1111/jep.13060

21. Rahm AK, Boggs JM, Martin C, Price DW, Beck A, Backer TE, Dearing JW. (2015). Facilitators and barriers to implementing screening, brief intervention, and referral to treatment (SBIRT) in primary care in integrated health care settings. Subst Abuse .2015;36(3):281--288. doi:10.1080/08897077.2014.951140

22. Sandelowski M. Whatever happened to qualitative description? Res Nurs Health. 2000;23(4):334-340.

23. Sandelowski M. What's in a name? Qualitative description revisited. Res Nurs Health. 2010;33(1):77-84. Doi:10.1002/nur.20362

24. Neergaard MA, Olesen F, Andersen RS, Sondergaard J. Qualitative description: The poor cousin of health research? BMC Med Res Methodol, 2009;9:52. Doi: 10.1186/1471-2288$9-52$

25. Chafe R. The value of qualitative description in health services and policy research. Healthcare Policy. 2017;12(3):12-18.

26. Tong A, Sainsbury P, Craig J. Consolidated criteria for reporting qualitative research (COREQ): A 32-item checklist for interviews and focus groups. Int J Qual Health C. 2007;19(6):349-357. 
27. Newhouse R, Janney M, Gilbert A, Agley J, Bakoyannis G, Ferren M, et al. Study protocol testing toolkit versus usual care for implementation of screening, brief intervention, and referral to treatment in hospitals: A phased cluster randomized approach. Addict Sci Clin Pract. 2018;13(28). doi: $10.1186 / \mathrm{s} 13722-018-0130-4$

28. Newhouse R, Thoele K, Agley J, \& Bakoyannis G. ERIC strategies improve screening for substance use in hospitals: A phased multi-site cluster randomized approach. Poster presented at AcademyHealth Annual Research Meeting; July 28-August 6, 2020; virtual.

29. Michie S, Johnston M, Abraham C, Lawton R, Parker D, Walker A. Making psychological theory useful for implementing evidence based practice: A consensus approach. Qual Saf Health Care. 2005;14(1): 26-33. doi:10.1136/qshc.2004.011155

30. Hsieh HF, Shannon SE. Three approaches to qualitative content analysis. Qual Health Res. 2005;15(9):1277-1288. doi:10.1177/1049732305276687

31. Saldaña J. The coding manual for qualitative researchers, $3^{\text {rd }}$ ed. Washington DC: Sage; 2015.

32. Miles MB, Huberman AM, Saldaña, J. Qualitative data analysis: A methods source book, $3^{\text {rd }}$ ed. Sage: Los Angeles, California; 2014.

33. Morse, J.M. Critical analysis of strategies for determining rigor in qualitative inquiry. Qual Health Res. 2015;25(9):1212-1222. doi: 10.1177/1049732315588501

34. Bernstein E, Bernstein J, Fernandez W, Hagan M, Mitchell P, Safi C, et al. An evidencebased alcohol screening, brief intervention and referral to treatment (SBIRT) curriculum for emergency department (ED) providers improves skill and utilization. Subst Abuse. 2007;28(4):79-92. doi:10.1300/J465v28n04_01. 
35. Hargraves D, White C, Frederick R, Cinibulk M, Peters M, Young A, Elder N.

Implementing SBIRT (screening, brief intervention and referral to treatment) in primary care: Lessons learned from a multi-practice evaluation portfolio. Pub Health Rev, 2017; 38:31. Doi: 10.1186/s40985-017-0077-0

36. Broyles LM, Rodriguez K, Kraemer KL, Sevick MA, Price PA, Gordon AJ. (2012b). A qualitative study of anticipated barriers and facilitators to the implementation of nurse-delivered alcohol screening, brief intervention, and referral to treatment for hospitalized patients in a Veterans Affairs medical center. Addict Sci Clin Pract. 2012;7:7. Doi: 10.1186/19400640-7-7 
Table 1. Participant Characteristics $(n=18)$

\begin{tabular}{lcc}
\hline \hline & Mean (Standard Deviation) & Range \\
\hline Age in years & $43.94(11.56)$ & $25-63$ \\
Years of registered nurse experience & $15.61(9.41)$ & $3-37$ \\
Years of experience on current unit & $8.94(8.23)$ & $1-32$ \\
\hline Sex & $\mathbf{n}$ & $\mathbf{\%}$ \\
Female & 17 & 94.44 \\
Male & 1 & 5.56 \\
Shift & & \\
Day & 13 & 72.22 \\
Night & 5 & 27.78 \\
Highest nursing degree & & \\
Associate's & 5 & 27.78 \\
Bachelor's & 12 & 66.67 \\
Master's & 1 & 5.56 \\
Doctoral & 0 & 0 \\
Training & & 22.22 \\
Screening only & 4 & 77.78 \\
Screening and brief intervention & 14 & \\
Type of facility of employment & & 27.78 \\
Academic health center & 5 & 22.22 \\
Community hospital & 4 & 50.00 \\
Critical access hospital & 9 & \\
\hline \hline
\end{tabular}


Table 2. Barriers and Facilitators to Implementation of SBIRT

\begin{tabular}{|c|c|c|}
\hline & Barriers & Facilitators \\
\hline $\begin{array}{l}\text { Nurse's } \\
\text { attitudes and } \\
\text { beliefs about } \\
\text { SBIRT }\end{array}$ & $\begin{array}{l}\text { 1. SBIRT one more thing to } \\
\text { do } \\
\text { 2. SBIRT long-term effects } \\
\text { not observable } \\
\text { 3. Screening questions } \\
\text { uncomfortable to ask } \\
\text { 4. Brief intervention not a } \\
\text { nursing function } \\
\text { 5. Acute care setting not best } \\
\text { setting to use SBIRT } \\
\text { 6. SBIRT doesn't provide new } \\
\text { information } \\
\text { 7. Feel unprepared to provide } \\
\text { intervention }\end{array}$ & $\begin{array}{l}\text { 1. Comfortable asking sensitive } \\
\text { questions and communicating } \\
\text { 2. SBIRT is an extension of } \\
\text { current skills } \\
\text { 3. SBIRT identifies need for } \\
\text { acute interventions } \\
\text { 4. SBIRT expands the nursing } \\
\text { role } \\
\text { 5. SBIRT is a useful tool } \\
\text { 6. The screening tool makes it } \\
\text { easier to ask routine questions } \\
\text { about substance use } \\
\text { 7. Knowledge of addiction and } \\
\text { how to use SBIRT } \\
\text { 8. SBIRT provides information } \\
\text { about resources after } \\
\text { hospitalization } \\
\text { 9. Screening is easy and doesn't } \\
\text { take long }\end{array}$ \\
\hline $\begin{array}{l}\text { Organizational } \\
\text { factors related } \\
\text { to SBIRT }\end{array}$ & $\begin{array}{l}\text { 1. Difficult to maintain } \\
\text { competency in brief } \\
\text { intervention } \\
\text { 2. No assessment of SBIRT or } \\
\text { feedback } \\
\text { 3. SBIRT not integrated into } \\
\text { the electronic medical } \\
\text { record or workflow } \\
\text { 4. Inadequate training } \\
\text { 5. Competing priorities } \\
\text { 6. Lack of adequate resources } \\
\text { in the community }\end{array}$ & $\begin{array}{l}\text { 1. SBIRT is incorporated into } \\
\text { routine practice } \\
\text { 2. SBIRT is an expectation of all } \\
\text { nurses } \\
\text { 3. Clear and streamlined process } \\
\text { for SBIRT } \\
\text { 4. Good training and ongoing } \\
\text { support } \\
\text { 5. SBIRT champion } \\
\text { 6. Ability to adapt SBIRT }\end{array}$ \\
\hline $\begin{array}{l}\text { Patients' } \\
\text { responses to } \\
\text { SBIRT }\end{array}$ & $\begin{array}{l}\text { 1. Hide substance use } \\
\text { 2. Not ready to change } \\
\text { behavior } \\
\text { 3. Reluctant to open up until } \\
\text { trusting nurse/patient } \\
\text { relationship established } \\
\text { 4. Feel angry or judged } \\
\text { 5. Difficult to use SBIRT if } \\
\text { patient is tired or in pain }\end{array}$ & $\begin{array}{l}\text { 1. Honest about substance use } \\
\text { 2. More effective when trusting } \\
\text { nurse/patient relationship has } \\
\text { been established } \\
\text { 3. Willing to change behavior } \\
\text { and accept available resources }\end{array}$ \\
\hline
\end{tabular}




\section{Appendix A: Semi-structured Interview Questions}

\section{Introductory Questions}

1. Tell me about how familiar you were with SBIRT before it was implemented on your unit.

2. What are your thoughts generally about the SBIRT process?

3. Tell me about the training you received in SBIRT.

4. Tell me how SBIRT was first introduced on your unit. What was the process that occurred? What did you think about SBIRT use when you first heard about it?

\section{Adoption: Decision to Use SBIRT}

5. Have you used SBIRT with patients?

**Note: identify which components of SBIRT (screening, brief intervention, referral to treatment) the participant uses in practice. Questions 6 through 13 should be tailored based on the participant's practice.

6. If yes - How did you decide to use SBIRT?

7. If no - How did you decide not to use SBIRT?

\section{Implementation: Use of SBIRT}

8. Tell me about a time you used SBIRT that you felt it went well?

9. Tell me about a time when you used SBIRT that it did not go well?

10. Describe what it is like for you to use SBIRT? How comfortable or confident do you feel using it?

11. What skills do you feel are important for nurses to have to use SBIRT? Describe your own skills using SBIRT.

12. What barriers have you run into using SBIRT? Tell me about a time if any when those barriers came into play.

13. What helps you use SBIRT? Tell me about a time if any when your use of SBIRT was facilitated by something or someone.

14. Overall have you found SBIRT to be a useful process?

\section{Sustainability and Spread}

15. Do you intend to continue to use SBIRT in your practice? Why or why not? 